\title{
Constraints on Chaplygin quartessence from the CLASS gravitational lens statistics and supernova data
}

\author{
Abha Dev ${ }^{1}$, Deepak Jain ${ }^{2}$, and J. S. Alcaniz ${ }^{3}$ \\ 1 Department of Physics and Astrophysics, University of Delhi, Delhi 110007, India \\ e-mail: abha@ducos.ernet.in \\ ${ }^{2}$ Deen Dayal Upadhyaya College, University of Delhi, Delhi 110015, India \\ e-mail: deepak@physics.du.ac.in \\ 3 Departamento de Física, Universidade Federal do Rio Grande do Norte, CP 1641, Natal - RN, 59072-970, Brasil
}

Received 4 November 2003 / Accepted 31 December 2003

\begin{abstract}
The nature of the dark components (dark matter and dark energy) that dominate the current cosmic evolution is a completely open question at present. In reality, we do not even know if they really constitute two separated substances. In this paper we use the recent Cosmic All Sky Survey (CLASS) lensing sample to test the predictions of one of the candidates for a unified dark matter/energy scenario, the so-called generalized Chaplygin gas $(\mathrm{Cg})$ which is parametrized by an equation of state $p=-A / \rho_{\mathrm{Cg}}^{\alpha}$ where $A$ and $\alpha$ are arbitrary constants. We show that, although the model is in good agreement with this radio source gravitational lensing sample, the limits obtained from CLASS statistics are only marginally compatible with the ones obtained from other cosmological tests. We also investigate the constraints on the free parameters of the model from a joint analysis between CLASS and supernova data.
\end{abstract}

Key words. cosmology: theory - cosmology: dark matter - cosmology: cosmological parameters - cosmology: distance scale

\section{Introduction}

As is well known, there is mounting observational evidence that our Universe is presently dominated by two exotic forms of matter or energy. Cold, nonbaryonic dark matter, which accounts for $\simeq 30 \%$ of the critical mass density and whose leading particle candidates are the axions and the neutralinos, was originally proposed to explain the general behavior of galactic rotation curves that differ significantly from the one predicted by Newtonian mechanics. Later on, it was also realized that the same concept is necessary for explaining the evolution of the observed structure in the Universe from density inhomogeneities of the size detected by a number of Cosmic Microwave Background (CMB) experiments. Dark energy or quintessence, which accounts for $\simeq 70 \%$ of the critical mass density and whose leading candidates are a cosmological constant $\Lambda$ and a relic scalar field $\phi$, has been inferred from a combination of astronomical observations which includes distance measurements of type Ia supernovae ( $\mathrm{SNe}$ Ia) indicating that the expansion of the Universe is speeding up not slowing down (Perlmutter et al. 1999; Riess et al. 1998), CMB anisotropy data suggesting $\Omega_{T} \simeq 1$ (de Bernardis et al. 2000; Spergel et al. 2003), and clustering estimates providing $\Omega_{m} \simeq 0.3$

Send offprint requests to: J. S. Alcaniz, e-mail: alcaniz@dfte.ufrn.br
(Calberg et al. 1996; Dekel et al. 1997). While the combination of the last two results implies the existence of a smooth component of energy that contributes with $\simeq 2 / 3$ of the critical density, the $\mathrm{SNe}$ Ia results require this component to have a negative pressure, which leads to a repulsive gravity.

Despite the good observational evidence for the existence of these two forms of energy, it has never been shown that in fact they constitute two separate substances. In this concern, some authors have proposed the so-called Unified Dark Matter/Energy scenarios (UDME) or quartessence, i.e., models in which these two dark components are seen as different manifestations of a single fluid (see, for instance, Matos \& Ureña-Lopez 2000; Davidson et al. 2001; Watterich 2002; Kasuya 2001; Padmanabhan \& Choudhury 2002). Among these theoretical proposals, an interesting attempt of unification was originally suggested by Kamenshchik et al. (2001) and developed by Bilić et al. (2002) and Bento et al. (2002), namely, the Chaplygin gas $(\mathrm{Cg})$, an exotic fluid whose equation of state is given by

$p_{\mathrm{Cg}}=-A / \rho_{\mathrm{Cg}}^{\alpha}$,

with $\alpha=1$ and $A$ a positive constant. In actual fact, the above equation for $\alpha \neq 1$ constitutes a generalization of the original Chaplygin gas equation of state proposed by Bento et al. (2002). The idea of a dark-matter-energy unification from an 
equation of state like Eq. (1) comes from the fact that the $\mathrm{Cg}$ can naturally interpolate between nonrelativistic matter $(p=0)$ and negative-pressure ( $p=-$ const.) dark energy regimes (see Bento et al. 2002; Alcaniz et al. 2002 for details).

Very recently, there has been a wave of interest in exploring theoretical (Bordemann \& Hoppe 1993; Hoppe 1993; Jackiw 2000; Gonzalez-Diaz 2003a,b; Kremer 2003; Khalatnikov 2003; Balakin et al. 2003; Bilic et al. 2003) and observational consequences of the Chaplygin gas, not only as a possibility of unification for dark matter/energy but also as a new candidate for dark energy only. These models have been tested for a number of cosmological data sets, including SNe Ia data (Fabris et al. 2002; Colistete et al. 2003; Avelino et al. 2003; Makler et al. 2003), statistical properties of gravitationally lensed quasars (Dev et al. 2003; Silva \& Bertolami 2003), CMB measurements (Bento et al. 2003a,b,c; Carturan \& Finelli 2002; Amendola et al. 2003), age and angular size - redshift tests (Alcaniz et al. 2002; Alcaniz \& Lima 2003), measurements of X-ray luminosity of galaxy clusters (Cunha et al. 2003), future lensing and SNe Ia experiments (Avelino et al. 2003; Silva \& Bertolami 2003; Sahni et al. 2003), as well as by observations of large scale structure (Multamaki et al. 2003; Bilic et al. 2003). The present situation is somewhat controversial, with some tests indicating good agreement between observational data and the theoretical predictions of the model and others ruling out the model as an actual possibility of description for our Universe (Sandvik et al. 2002; Bean \& Dore 2003) (see, however, Beça et al. 2003).

The aim of the current paper is to check the validity of such models with radio-selected gravitational lens statistics and also with a combination of gravitational lensing and SNe Ia data. To do so, we adopt the most recent radio source gravitational lensing sample, namely, the Cosmic All Sky Survey (CLASS) statistical data which consists of 8958 radio sources out of which 13 sources are multiply imaged (Browne et al. 2002; Chae et al. 2002). Here, however, we work only with those multiply imaged sources whose image-splittings are known (or likely) to be caused by single galaxies, which reduces the total number of lenses to 9. For the cosmological background we consider a flat scenario in which the generalized $\mathrm{Cg}$ together with the observed baryonic content are responsible for the dynamics of the present-day Universe (UDME or quartessence models). In our computations we adopt $\Omega_{\mathrm{b}}=0.04$, in accordance with the latest measurements of the Hubble parameter (Freedman et al. 2002) and of the baryon density at nucleosynthesis (Burles et al. 2001).

This paper is organized as follows. In Sect. 2 we present the distance formulae necessary to our analysis. In Sect. 3 we discuss the CLASS statistical sample, especially the observational criteria used as well as the restrictions adopted in our analysis. In Sect. 4, we derive the corresponding limits on $\mathrm{Cg}$ scenarios from CLASS lensing statistics. We also examine the constraints obtained from the statistical combination of lensing data with recent SNe Ia observations and compare our constraints with others derived from independent analyses. Finally, in Sect. 5, we finish the paper by summarizing its main results.

\section{Basic equations}

By inserting Eq. (1) into the energy conservation law, one finds,

$\rho_{\mathrm{Cg}}=\left[A+B\left(\frac{R_{o}}{R}\right)^{3(1+\alpha)}\right]^{\frac{1}{1+\alpha}}$

or, equivalently,

$\rho_{\mathrm{Cg}}=\rho_{\mathrm{Cg}_{o}}\left[A_{\mathrm{s}}+\left(1-A_{\mathrm{s}}\right)\left(\frac{R_{o}}{R}\right)^{3(1+\alpha)}\right]^{\frac{1}{1+\alpha}}$,

where $\rho_{\mathrm{Cg}}$ stands for the Cg energy density, the subscript $o$ denotes present day quantities, $R(t)$ is the cosmological scale factor, $B=\rho_{\mathrm{Cg}_{o}}^{1+\alpha}-A$ is a constant and $A_{\mathrm{s}}=A / \rho_{\mathrm{Cg}_{o}}^{1+\alpha}$ is a quantity related to the sound speed of the Chaplygin gas today $\left(v_{\mathrm{s}}^{2}=\alpha A_{\mathrm{s}}\right)$.

A fundamental quantity related to the observables here considered is the distance -redshift relation, given by

$\chi=\frac{c}{R_{o} H_{o}} \int_{(1+z)^{-1}}^{1} \frac{\mathrm{d} x}{x^{2} E\left(\Omega_{\mathrm{b}}, A_{\mathrm{s}}, \alpha, x\right)}$,

where $x=\frac{R(t)}{R_{o}}=(1+z)^{-1}$ is a convenient integration variable, $\Omega_{\mathrm{b}}$ stands for the baryonic matter density parameter, and the dimensionless function $E\left(\Omega_{\mathrm{b}}, A_{\mathrm{s}}, \alpha, x\right)$ is written as

$E=\left\{\frac{\Omega_{\mathrm{b}}}{x^{3}}+\left(1-\Omega_{\mathrm{b}}\right)\left[A_{\mathrm{s}}+\frac{\left(1-A_{\mathrm{s}}\right)}{x^{3(\alpha+1)}}\right]^{\frac{1}{\alpha+1}}\right\}^{1 / 2}$.

For the lensing statistics developed in the next section, two concepts are of fundamental importance, namely, the angular diameter distance, $D_{\mathrm{LS}}\left(z_{\mathrm{L}}, z_{\mathrm{S}}\right)=\frac{R_{o} r_{1}\left(z_{\mathrm{L}}, z_{\mathrm{S}}\right)}{\left(1+z_{\mathrm{S}}\right)}$, between two objects, for example a lens at $z_{\mathrm{L}}$ and a source (galaxy) at $z_{\mathrm{S}}$,

$D_{\mathrm{LS}}\left(z_{\mathrm{L}}, z_{\mathrm{S}}\right)=\frac{c H_{o}^{-1}}{\left(1+z_{\mathrm{S}}\right)} \times \int_{x_{\mathrm{S}}^{\prime}}^{x_{\mathrm{L}}^{\prime}} \frac{\mathrm{d} x}{x^{2} E\left(\Omega_{\mathrm{b}}, A_{\mathrm{s}}, \alpha, x\right)}$.

and the age-redshift relation,

$t_{z}=\frac{1}{H_{o}} \int_{0}^{(1+z)^{-1}} \frac{\mathrm{d} x}{x E\left(\Omega_{\mathrm{b}}, A_{\mathrm{s}}, \alpha, x\right)}$.

From the above equations, we note that UDME models reduce to the $\Lambda \mathrm{CDM}$ case for $\alpha=0$ whereas the standard Einstein-de Sitter behavior is fully recovered for $A_{\mathrm{s}}=0$ (see also Fabris et al. 2003; Avelino et al. 2003).

\section{Gravitational lensing statistics of the CLASS sample}

Gravitational lensing directly probes the mass distribution in the Universe so that an investigation of lensing events of sources at high redshifts can provide important information about the global cosmological parameters and the structure of the Universe. The use of gravitational lensing statistics as a cosmological tool was first considered in detail by Turner et al. (1984). Subsequently, it was realized that a comparison of theoretical lensing probabilities with gravitational lensing observations provided an efficient constraint on the cosmological 
constant (Fukugita et al. 1990; Turner 1990; Fukugita et al. 1992; Kochanek 1996) or more generally on the density and the equation of state of the dark energy component (Zhu 1998; 2000a,b; Sarbu et al. 2001; Chae et al. 2002; Huterer \& Ma 2003). However, the absence of an unbiased statistical sample of sources that is complete to within well-defined observational selection criteria and the uncertainties in the luminosity function (LF) of galaxies have seriously complicated the application of such methods.

\subsection{The CLASS statistical sample}

Recently, the CLASS collaboration ${ }^{1}$ reported the so far largest lensing sample suitable for statistical analysis, in which 13 out of the 8958 radio sources are multiply imaged (Myers et al. 2002; Browne et al. 2002). This sample is well defined through the following observational selection criteria (Myers et al. 2002; Chae 2002; Browne et al. 2002): (i) the spectral index between $1.4 \mathrm{GHz}$ and $5 \mathrm{GHz}$ is flatter than -0.5 , i.e., $\alpha \geq-0.5$ with $S_{v} \propto v^{\alpha}$, where $S_{v}$ is the flux density measured in milli-jansky; (ii) the total flux density of each source is $\geq 20 \mathrm{mJy}$ at $8.4 \mathrm{GHz}$; (iii) the total flux density of each source is $\geq S^{0} \equiv 30 \mathrm{mJy}$ at $5 \mathrm{GHz}$; (iv) the image components in lens systems must have separations $\geq 0.3$ arcsec. The sources probed by CLASS at $v=5 \mathrm{GHz}$ are well represented by a power-law differential number-flux density relation: $|\mathrm{d} N / \mathrm{d} S| \propto\left(S / S^{0}\right)^{\eta}$ with $\eta=2.07 \pm 0.02(1.97 \pm 0.14)$ for $S \geq S^{0}\left(\leq S^{0}\right)$. The redshift distribution of unlensed sources in the sample is adequately described by a Gaussian model with a mean redshift $z=1.27$ and dispersion of 0.95 (Chae 2002). Guided by the above information about (i) the number-flux density relation and (ii) the redshift distribution of unlensed sources, we simulate the unlensed radio sources ( 8945 in number) of the CLASS statistical sample using the Monte-Carlo technique (rejection method).

In this paper, following Dev et al. (2003), we work only with those multiply imaged sources whose image-splittings are known (or likely) to be caused by single galaxies. This means that our database is constituted by 9 lenses out of a sample of 8954 radio sources.

\subsection{Lensing statistics}

We start our analysis by assuming the singular isothermal sphere (SIS) model for the lens mass distribution. As has been discussed elsewhere this assumption is a good approximation to the real mass distribution in galaxies (see, e.g., Turner et al. 1984). In this case, the cross-section for lensing events is given by

$\sigma_{\mathrm{SIS}}=16 \pi^{3}\left(\frac{v}{c}\right)^{4}\left(\frac{D_{\mathrm{OL}} D_{\mathrm{LS}}}{D_{\mathrm{OS}}}\right)^{2}$

where $v$ represents the velocity dispersion and $D_{\mathrm{OL}}, D_{\mathrm{OS}}$ and $D_{\mathrm{LS}}$ are, respectively, the angular diameter distances from the observer to the lens, from the observer to the source and

\footnotetext{
1 The Cosmic Lens All Sky Survey (CLASS): http://www. aoc.nrao.edu/ smyers/class.html
}

between the lens and the source. By ignoring evolution of the number density of galaxies and assuming that the comoving number density is conserved, the differential probability of a lensing event can be expressed as

$\mathrm{d} \tau=n_{o}\left(1+z_{\mathrm{L}}\right)^{3} \sigma_{\mathrm{SIS}} \frac{c \mathrm{~d} t}{\mathrm{~d} z_{\mathrm{L}}} \mathrm{d} z_{\mathrm{L}}$

where the quantity $\mathrm{d} t / \mathrm{d} z_{\mathrm{L}}$ can be easily obtained from Eq. (7) and the present-day comoving number density of galaxies is

$n_{o}=\int_{0}^{\infty} \phi(L) \mathrm{d} L$.

The differential optical depth of lensing in traversing $\mathrm{d} z_{\mathrm{L}}$ with angular separation between $\phi$ and $\phi+\mathrm{d} \phi$ is (Fukugita et al. 1990; Turner 1990; Fukugita et al. 1992):

$\frac{\mathrm{d}^{2} \tau}{\mathrm{d} z_{\mathrm{L}} \mathrm{d} \phi} \mathrm{d} \phi \mathrm{d} z_{\mathrm{L}}=F^{*}\left(1+z_{\mathrm{L}}\right)^{3}\left(\frac{D_{\mathrm{OL}} D_{\mathrm{LS}}}{R_{o} D_{\mathrm{OS}}}\right)^{2} \frac{1}{R_{o}} \frac{c \mathrm{~d} t}{\mathrm{~d} z_{\mathrm{L}}}$
$\times \frac{\gamma / 2}{\Gamma\left(\alpha+1+\frac{4}{\gamma}\right)}\left(\frac{D_{\mathrm{OS}}}{D_{\mathrm{LS}}} \phi\right)^{\frac{\gamma}{2}\left(\alpha+1+\frac{4}{\gamma}\right)} \times \exp \left[-\left(\frac{D_{\mathrm{OS}}}{D_{\mathrm{LS}}} \phi\right)^{\frac{\gamma}{2}}\right] \frac{\mathrm{d} \phi}{\phi} \mathrm{d} z_{\mathrm{L}}$

where the function $F^{*}$ is defined as

$F^{*}=\frac{16 \pi^{3}}{c H_{0}^{3}} \phi_{*} v_{*}^{4} \Gamma\left(\alpha+\frac{4}{\gamma}+1\right)$.

In Eq. (10), $\phi(L)$ is the Schechter LF (Schechter 1976) given by

$\phi(L) \mathrm{d} L=\phi_{*}\left(\frac{L}{L_{*}}\right)^{\alpha} \exp \left(-L / L_{*}\right) \frac{\mathrm{d} L}{L_{*}}$.

In order to relate the characteristic luminosity $L_{*}$ to the characteristic velocity dispersion $v_{*}$, we use the Faber-Jackson relation (Faber \& Jackson 1976) for E/S0 galaxies $\left(L_{*} \propto v_{*}^{\gamma}\right)$, with $\gamma=4$. For the analysis presented here we neglect the contribution of spirals as lenses because their velocity dispersion is small when compared to ellipticals.

The two large-scale galaxy surveys, namely, the $2 \mathrm{dFGRS}^{2}$ and the SDSS $^{3}$ have produced converging results on the total LF. The surveys determined the Schechter parameters for galaxies (all types) at $z \leq 0.2$. Chae (Chae 2002) has worked extensively on the information provided by these recent galaxy surveys to extract the local type-specific LFs. For our analysis here, we adopt the normalization-corrected Schechter parameters of the 2dFGRS survey (Folkes et al. 1999): $\alpha=-0.74$, $\phi^{*}=0.82 \times 10^{-2} h^{3} \mathrm{Mpc}^{-3}, v^{*}=185 \mathrm{~km} \mathrm{~s}^{-1}$ and $F^{*}=0.014$.

The normalized image angular separation distribution for a source at $z_{\mathrm{S}}$ is obtained by integrating $\frac{\mathrm{d}^{2} \tau}{\mathrm{d} z_{\mathrm{L}} \mathrm{d} \phi}$ over $z_{\mathrm{L}}$ :

$\frac{\mathrm{d} \mathcal{P}}{\mathrm{d} \phi}=\frac{1}{\tau\left(z_{\mathrm{S}}\right)} \int_{0}^{z_{\mathrm{S}}} \frac{\mathrm{d}^{2} \tau}{\mathrm{d} z_{\mathrm{L}} \mathrm{d} \phi} \mathrm{d} z_{\mathrm{L}}$.

The corrected (for magnification and selection effects) image separation distribution function for a single source at redshift $z_{\mathrm{S}}$

\footnotetext{
2 The 2dF Galaxy RedshiftSurvey (2dfGRS):

http://msowww. anu.edu.au/2dFGRS/

3 Sloan Digital Sky Survey: http://www . sdss . org/
} 
is given by (Kochanek 1996; Chiba \& Yoshii 1999)

$$
\begin{aligned}
P^{\prime}(\Delta \theta)= & \mathcal{B} \frac{\gamma}{2 \Delta \theta} \int_{0}^{z \mathrm{~s}}\left[\frac{D_{0 \mathrm{~S}}}{D_{\mathrm{LS}}} \phi\right]^{\frac{\gamma}{2}\left(\alpha+1+\frac{4}{\gamma}\right)} \\
& \times F^{*} \frac{c \mathrm{~d} t}{\mathrm{~d} z_{\mathrm{L}}} \times \exp \left[-\left(\frac{D_{0 \mathrm{~S}}}{D_{\mathrm{LS}}} \phi\right)^{\frac{\gamma}{2}}\right] \frac{\left(1+z_{\mathrm{L}}\right)^{3}}{\Gamma\left(\alpha+\frac{4}{\gamma}+1\right)} \\
& \times\left[\left(\frac{D_{\mathrm{OL}} D_{\mathrm{LS}}}{R_{0} D_{\mathrm{OS}}}\right)^{2} \frac{1}{R_{0}}\right] \mathrm{d} z_{\mathrm{L}}
\end{aligned}
$$

Similarly, the corrected lensing probability for a given source at redshift $z$ is given by

$$
P^{\prime}=\tau\left(z_{\mathrm{S}}\right) \int \frac{\mathrm{d} \mathcal{P}}{\mathrm{d} \phi} \mathcal{B} \mathrm{d} \phi .
$$

Here $\phi$ and $\Delta \theta$ are related as $\phi=\frac{\Delta \theta}{8 \pi\left(v^{*} / c\right)^{2}}$, and $\mathcal{B}$ is the magnification bias. This is taken into account because, as widely known, gravitational lensing causes a magnification of images and this transfers the lensed sources to higher flux density bins. In other words, the lensed sources are over-represented in a flux-limited sample. The magnification bias $\mathcal{B}\left(z_{\mathrm{S}}, S_{v}\right)$ increases the lensing probability significantly in a bin of total flux density $\left(S_{v}\right)$ by a factor

$$
\begin{aligned}
\mathcal{B}\left(z_{\mathrm{S}}, S_{v}\right)= & \left|\frac{\mathrm{d} N_{z_{\mathrm{S}}}\left(>S_{v}\right)}{\mathrm{d} S_{v}}\right|^{-1} \\
& \times \int_{\mu_{\min }}^{\mu_{\max }}\left|\frac{\mathrm{d} N_{z_{\mathrm{S}}}\left(>S_{v} / \mu\right)}{\mathrm{d} S_{v}} p(\mu)\right| \frac{1}{\mu} \mathrm{d} \mu .
\end{aligned}
$$

In the above expression $N_{z_{\mathrm{S}}}\left(>S_{v}\right)$ is the intrinsic flux density relation for the source population at redshift $z_{\mathrm{S}} . N_{z_{\mathrm{S}}}\left(>S_{v}\right)$ gives the number of sources at redshift $z_{S}$ having flux greater than $S_{v}$. For the SIS model, the magnification probability distribution is $p(\mu)=8 / \mu^{3}$. The minimum and maximum total magnifications $\mu_{\min }$ and $\mu_{\max }$ in Eq. (17) depend on the observational characteristics as well as on the lens model. For the SIS model, the minimum total magnification is $\mu_{\min } \simeq 2$ and the maximum total magnification is $\mu_{\max }=\infty$. The magnification bias $\mathcal{B}$ depends on the differential number-flux density relation $\left|\mathrm{d} N_{z \mathrm{~S}}\left(>S_{v}\right) / \mathrm{d} S_{v}\right|$. The differential number-flux relation needs to be known as a function of the source redshift. At present, redshifts of only a few CLASS sources are known. We, therefore, ignore redshift dependence of the differential number-flux density relation. Following Chae (2002), we further ignore the dependence of the differential number-flux density relation on the spectral index of the source.

An important selection criterion for the CLASS statistical sample is that the ratio of the flux densities of the fainter to the brighter images $\mathcal{R}_{\min }$ is $\geq 0.1$. Given such an observational limit,the minimum total magnification for double imaging for the adopted model of the lens is (Chae 2002):

$\mu_{\min }=2 \frac{1+\mathcal{R}_{\min }}{1-\mathcal{R}_{\min }}$

Another selection criterion is that the image components in lens systems must have separations $\geq 0.3$ arcsec. We incorporate this selection criterion by setting the lower limit of $\Delta \theta$ in Eq. (16) as 0.3 arcsec.

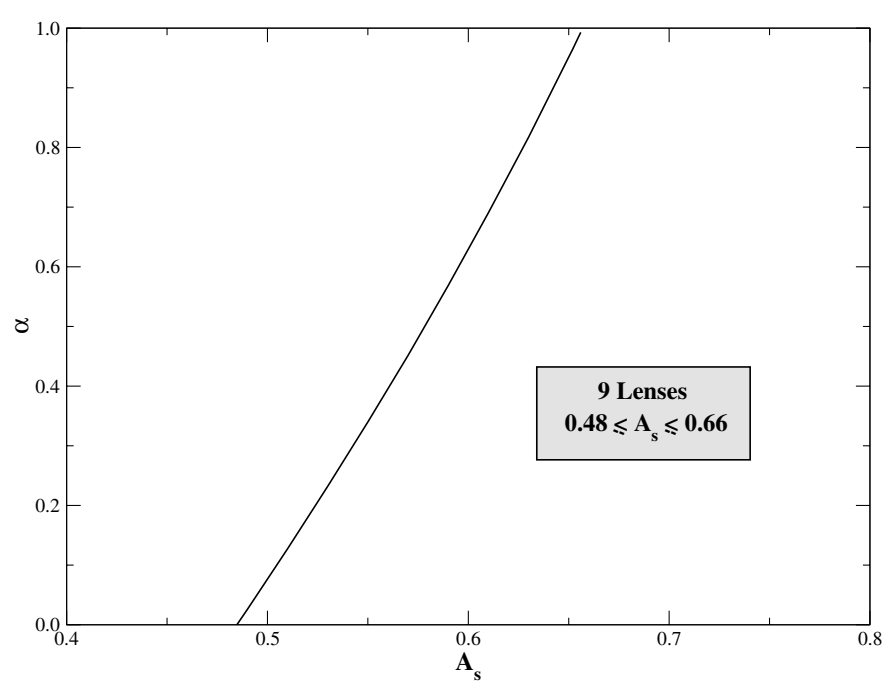

Fig. 1. Contour for 9 lensed radio sources in the parametric space $A_{\mathrm{s}}-$ $\alpha$. While the entire range of $\alpha$ is allowed, the parameter $A_{\mathrm{s}}$ is restricted to the interval $0.48 \leq A_{\mathrm{s}} \leq 0.66$.

\section{Testing $\mathrm{Cg}$ scenarios against observations}

The expected number of lensed radio sources is $N_{\text {lens }}=\sum P_{i}^{\prime}$, where $P_{i}^{\prime}$ is the lensing probability of the $i$ th source and the sum is over the entire adopted sample. The expected number of lensed sources is thus a function of the parameters $A_{\mathrm{s}}$ and $\alpha$. We have done a grid search for those combinations $\left(A_{\mathrm{s}}, \alpha\right)$ by fixing $N_{\text {lens }}=9$. In Fig. 1 we show the contour for 9 lensed radio sources in the parametric space $A_{\mathrm{s}}-\alpha$. As can be seen, while the entire range of $\alpha$ is allowed, the parameter $A_{\mathrm{s}}$ is tightly restricted to the interval $0.48 \leq A_{\mathrm{s}} \leq 0.66$. This particular range for $A_{\mathrm{s}}$ is not compatible with the one obtained from a SNe analysis involving 92 events of the Supernova Cosmology Project and High- $z$ supernova Search Team, i.e., $A_{\mathrm{s}}>0.69$ at $95 \%$ confidence level (Avelino et al. 2003) and is only marginally compatible with the limits from age $+\mathrm{SNe}$ performed by Makler et al. (2003). A comparison between the above interval with the one restricted by age estimates of high- $z$ objects shows that the $N_{\text {lens }}$ test for the CLASS sample is compatible with the existence of the radio galaxy LBDS 53W091 (3.5 Gyr at $z=1.55$ ) which implies $A_{\mathrm{s}} \geq 0.52$ but that it is not in accordance with the existence of the 4.0-Gyr-old radio galaxy 53W069 (at $z=1.43$ ) and the 2.0-Gyr-old quasar APM 08279+5255 (at $z=3.91$ ) which requires, respectively, $A_{\mathrm{s}} \geq 0.72$ and $A_{\mathrm{s}} \geq 0.82$ (Alcaniz et al. 2003). The above interval from the $N_{\text {lens }}$ test is also not in agreement with the tight limits obtained by Silva \& Bertolami (2003) from future $\mathrm{SNe}$ and lensing data, i.e., $0.75 \leq A_{\mathrm{s}} \leq 0.79$ at $2 \sigma(\alpha \lesssim 0.2)$.

The likelihood function for lensing can be written as

$\mathcal{L}=\prod_{i=1}^{N_{U}}\left(1-P_{i}^{\prime}\right) \prod_{k=1}^{N_{L}} P^{\prime}(\Delta \theta)$.

Here $N_{L}$ is the observed number of multiple-imaged lensed radio sources and $N_{U}$ is the number of unlensed sources in the adopted sample. The results of our analysis for the $\mathrm{Cg}$ model are displayed in Fig. 2a. The contours correspond to the $68.3 \%$ and $95.4 \%$ confidence level $(\mathrm{cl})$ in the $\left(A_{\mathrm{s}}, \alpha\right)$ plane. Although 

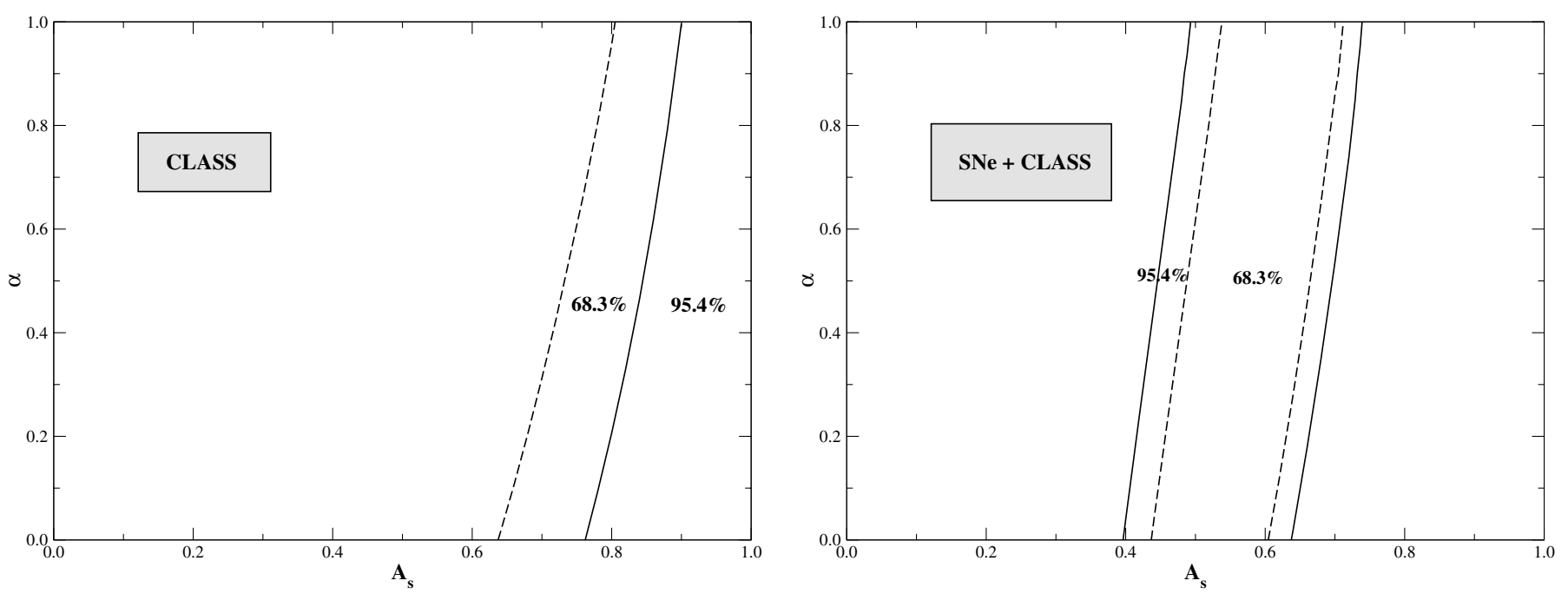

Fig. 2. a) Confidence regions in the plane $A_{\mathrm{s}}-\alpha$ arising from CLASS lensing statistics. Dashed and solid lines indicate contours of constant likelihood at $68.3 \%$ and $95.4 \%$ confidence levels, respectively. b) The likelihood contours in the $\alpha-A_{\mathrm{s}}$ plane for the joint lensing $+\mathrm{SNe}$ Ia analysis described in the text.

the entire range of $\alpha$ is permissible within the $68.3 \%$ confidence level, the parameter $A_{\mathrm{s}}$ is constrained to be $\leq 0.8$ at $68.3 \% \mathrm{cl}$ and $\leq 0.9$ at $95.4 \% \mathrm{cl}$. For this analysis the best fit model occurs for $A_{\mathrm{s}}=0.36$ and $\alpha=0.35$, which corresponds to an accelerating scenario with a deceleration parameter $q_{o}=-0.02$ and a total expanding age of $7.33 \mathrm{~h}^{-1}$ Gyr. Although not very restrictive, the constraints on the parameter $A_{\mathrm{s}}$ from the CLASS lensing sample of radio sources are more stringent than those obtained from the optical gravitational lensing surveys for quasars (Dev et al. 2003). However, it still limits the parameter $\alpha$ very weakly.

\subsection{Joint analysis with supernova data}

In order to perform a joint analysis with CLASS and SNe data sets, we first follow the conventional magnitude-redshift test (see, for example, Goliath et al. 2001; Dicus \& Repko 2003; Padmanabhan \& Choudhury 2003) and use the SNe Ia data set that corresponds to the primary fit $\mathrm{C}$ of Perlmutter et al. (1999) together with the highest redshift supernova observed so far, i.e., the $1997 \mathrm{ff}$ at $z=1.755$ and effective magnitude $m^{\text {eff }}=26.02 \pm 0.34$ (Benitez et al. 2002) and two newly discovered SNe Ia, namely, SN 2002dc at $z=0.475$ and $m^{\text {eff }}=22.73 \pm 0.23$ and SN 2002dd at $z=0.95$ and $m^{\text {eff }}=24.68 \pm 0.2$ (Blakeslee et al. 2003). We thus work with a total of 57 supernovae. The apparent magnitude of a given $\mathrm{SNe}$ is related to the luminosity distance $d_{\mathrm{L}}$ by the known relation $m=\mathcal{M}+5 \log D_{\mathrm{L}}$, where $D_{\mathrm{L}}=H_{0} d_{\mathrm{L}}$ and $\mathcal{M}$ is given by the intercept obtained by fitting the low-redshift data set to $m(z)=\mathcal{M}+5 \log (c z)$ (Hamuy et al. 1996). The value obtained is $\mathcal{M}=-3.325$ and confirms the results of Perlmutter et al. (1999). For the sake of completeness, we perform such $\mathrm{SNe}$ analysis. The best fit model occurs for $A_{\mathrm{s}}=0.52$ and $\alpha=-0.2$ with a minimum value of $\chi_{\text {min }}^{2}=64.31$ (which corresponds to $\chi_{v}^{2}=1.17$ ). When this magnitude-redshift test is combined with CLASS lensing statistics, tighter constraints on the $A_{\mathrm{s}}$ parameter can be obtained. As was shown, the index $\alpha$ is highly insensitive to SNe Ia data (Makler et al. 2003). Figure 2b shows the result of this joint analysis. For the combined $\chi^{2}$ analysis we used $\chi_{\text {total }}^{2}=\chi_{\mathrm{SNe}}^{2}-2 \ln l$, where $l=\mathcal{L}_{\text {lens }} / \mathcal{L}_{\max }^{\text {lens }}$ is the normalized likelihood for lenses. As can be seen, the limits on $A_{\mathrm{s}}$ are more restrictive now than those imposed by the gravitational lensing statistics of the CLASS sample (Fig. 2a). Within $68.3 \% \mathrm{cl}$, the constraints on the parameter $A_{\mathrm{s}}$ are as follows: $0.39 \leq A_{\mathrm{s}} \leq 0.71$ at $68.3 \% \mathrm{cl}$ and $0.35 \leq A_{\mathrm{s}} \leq 0.74$ at $95.4 \%$ cl. In particular, the best fit model occurs for $A_{\mathrm{s}}=0.58$ and $\alpha=0.5$, corresponding to a $7.93 h^{-1}$-Gyr-old, accelerating Universe with a deceleration parameter $q_{o}=-0.33$.

\section{Conclusion}

A considerable amount of observational evidence suggests that the current evolution of our Universe is fully dominated by two dark components, the so-called dark matter and dark energy. The nature of these components, however, is a tantalizing mystery at present, and it is not even known if they constitute two separate substances. In this paper we have investigate some observational predictions of cosmologies driven by an exotic component named the generalized Chaplygin gas. These models constitute an interesting possibility of unification for dark matter/energy, where these two dark components are seen as different manifestations of a single fluid (UDME). We have investigated observational constraints from lensing statistics on spatially flat UDME scenarios. Since gravitational lensing statistics constitutes an independent way of constraining cosmological parameters we have used the most recent lensing data, namely, the Cosmic All Sky Survey (CLASS) sample to obtain the $68.3 \%$ and $95.4 \%$ confidence intervals on the parameters of the $\mathrm{Cg}$ equation of state. Our statistical analysis shows that the best fit scenario for these data occurs at $A_{\mathrm{s}}=0.36$ and $\alpha=0.35$. At $68.3 \% \mathrm{cl}$, parameter $A_{\mathrm{s}}$ is restricted to $\leq 0.8$ while the entire range of $\alpha$ is allowed. By considering the observed number of lensed radio galaxies we tightly constrain $A_{\mathrm{s}}$ to the interval $0.48 \leq A_{\mathrm{s}} \leq 0.66$. From a joint $\chi^{2}$ analysis with $\mathrm{SNe}$ 
Ia data we obtain $0.35 \leq A_{\mathrm{s}} \leq 0.74$ at $95.4 \%$ cl with the best fit model occurring for $A_{\mathrm{s}}=0.58$ and $\alpha=0.5$, which corresponds to an accelerating scenario with a deceleration parameter $q_{o}=-0.33$ and a total expanding age of $7.93 h^{-1}$ Gyr. As has been commented earlier, such results are only marginally consistent with those obtained from independent cosmological tests. It means that only with a more general analysis, possibly a joint investigation involving different classes of cosmological data, it will be possible to delimit the $A_{\mathrm{s}}-\alpha$ plane more precisely, as well as to test more properly the consistency of these scenarios as a viable possibility of unification for the dark matter and dark energy scenarios.

Acknowledgements. The authors are very grateful to Zong-Hong Zhu and R. Silva for many valuable discussions and a critical reading of the manuscript. JSA is supported by the Conselho Nacional de Desenvolvimento Científico e Tecnológico (CNPq - Brasil) and CNPq(62.0053/01-1-PADCT III/Milenio).

\section{References}

Alcaniz, J. S., Jain, D., \& Dev, A. 2003, Phys. Rev. D, 67, 043514 Alcaniz, J. S., \& Lima, J. A. S. 2003 [astro-ph/0308465]

Amendola, L., Finelli, F., Burigana, C., \& Carturan, D. 2003, JCAP, 0307, 5

Avelino, P. P., Beça, L. M. G., de Carvalho, J. P. M., Martins, C. J. A. P. 2003 [astro-ph/0307427]

Avelino, P. P., Beça, L. M. G., de Carvalho, J. P. M., Martins, C. J. A. P., \& Pinto, P. 2003, Phys. Rev. D, 67, 023511

Balakin, A. B., Pavon, D., Schwarz, D. J., \& Zimdahl, W. 2003, [astro-ph/0302150]

Bean, R., \& Dore, O. 2003, Phys. Rev. D, 68, 023515

Beça, L. M. G., Avelino, P. P., de Carvalho, J. P. M., \& Martins, C. J. A. P. 2003, Phys. Rev. D, 67, 101301(R)

Benitez, N., Riess, A. G., Nugent, P. E., et al. 2002, ApJ, 577, L1

Bento, M. C., Bertolami, O., \& Sen, A. A. 2002, Phys. Rev. D, 66, 043507

Bento, M. C., Bertolami, O., \& Sen, A. A. 2003a, Phys. Rev. D, 67, 063003

Bento, M. C., Bertolami, O., \& Sen, A. A. 2003b [astro-ph/0303538]

Bento, M. C., Bertolami, O., \& Sen, A. A. 2003c [gr-qc/0305086]

Bilić, N., Tupper, G. B., \& Viollier, R. D. 2002, Phys. Lett. B, 535, 17

Bilic, N., Lindebaum, R. J., Tupper, G. B., \& Viollier, R. D. 2003 [astro-ph/0310181]

Bilic, N., Lindebaum, R. J., Tupper, G. B., \& Viollier, R. D. 2003 [astro-ph/0307214]

Blakeslee, J. P., Tsvetanov, Z. I., Riess, A. G., et al. 2003, ApJ, 589, 693

Bordemann, M., \& Hoppe, J. 1993, Phys. Lett. B, 317, 315

Browne, I. W. A., et al. 2002, MNRAS, in press [astro-ph/0211069]

Burles, S., Nollett, K. M., \& Turner, M. S. 2001, ApJ, 552, L1

Calberg, R. G., Yee, H. K. C., Ellingson, E., et al. 1996, ApJ, 462, 32

Carturan, D., \& Finelli, F. 2002 [astro-ph/0211626]

Chae, K.-H., Biggs, A. D., Blandford, R. D., et al. 2002, Phys. Rev. Lett., 89, 151301

Chae, K.-H. 2002 [astro-ph/0211244]

Chiba, M., \& Yoshii, Y. 2001, ApJ, 510, 42
Colistete Jr., R., Fabris, J. C., Gonçalves, S. V. B., de Souza, P. E. 2003 [astro-ph/0303338]

Cunha, J. V., Lima, J. A. S., \& Alcaniz, J. S. 2003 [astro-ph/0306319]

Davidson, A., Karasik, D., \& Lederer, Y. 2001 [gr-qc/0111107]

de Bernardis, P., Ade, P. A. R., Bock, J. J., et al. 2000, Nature, 404, 955

Dekel, A., Burstein, D., \& White, S. 1997, in Critical Dialogues in Cosmology, ed. N. Turok (Singapore: World Scientific)

Dev, A., Alcaniz, J. S., \& Jain, D. 2003, Phys. Rev. D, 67, 023515

Dev, A., Jain, D., \& Mahajan, S. 2003 [astro-ph/0307441]

Dicus, D. A., \& Repko, W. W., Phys. Rev. D, 67, 083520

Faber, S. M., \& Jackson, R. E. 1976, ApJ, 204, 668

Fabris, J. C., Goncalves, S. V. B., \& de Souza, P. E. 2002 [astro-ph/0207430]

Fabris, J. C., Goncalves, S. V. B., \& de Sá Ribeiro, R. 2003 [astro-ph/0307028]

Folkes, S., Ronen, S., Price, I., et al. 1999, MNRAS, 308, 459

Freedman, W. L., Madore, B. F., Gibson, B. K., et al. 2001, ApJ, 553, 47

Fukugita, M., Futamase, T., \& Kasai, M. 1990, MNRAS, 246, 24

Fukugita, M., Futamase, T., Kasai, M., \& Turner, E. L., ApJ, 393, 3

Goliath, M., Amanullah, R., Astier, P., Goobar, A., \& Pain, R. 2001, A\&A, 380, 6

Gonzalez-Diaz, P. F. 2003a, Phys. Rev. D, 68, 021303

Gonzalez-Diaz, P. F. 2003b, Phys. Lett. B, 562, 1

Hamuy, M., Phillips, M. M., Suntzeff, N. B., et al. 1996, AJ, 112, 2391

Hoppe, J. 1993 [hep-th/9311059]

Huterer, D., \& Ma, C.-P. 2003 [astro-ph/0307301]

Jackiw, R. 2000 [physics/0010042]

Kasuya, S. 2001, Phys. Lett. B, 515, 121

Kamensshchik, A., Moschella, U., \& Pasquier, V. 2001, Phys. Lett. B, 511,265

Khalatnikov, I. M. 2003, Phys. Lett. B, 563, 123

Kochanek, C. S. 1996, ApJ, 466, 638

Kremer, G. M. 2003, Gen. Rel. Grav., 35, 1459

Makler, M., de Oliveira, S. Q., \& Waga, I. 2003, Phys. Lett. B, 555, 1

Matos, T., \& Ureña-Lopez, L. A. 2000, Class. Quantum Grav., 17, L75

Matos, T., \& Ureña-Lopez, L. A. 2001, Phys. Rev. D, 63, 063506

Multamaki, T., Manera, M., \& Gaztanaga, E. 2003 [astro-ph/0307533]

Myers, S. T., et al. 2002, MNRAS, in press [astro-ph/0211073]

Padmanabhan, T., \& Choudhury, T. R. 2003, MNRAS, 344, 823

Padmanabhan, T., \& Choudhury, T. R. 2002, Phys. Rev. D, 66, 081301

Perlmutter, S., Aldering, G., Goldhaber, G., et al. 1999, ApJ, 517, 565

Riess, A., Filippenko, A. V., Challis, P., et al. 1998, AJ, 116, 1009

Sahni, V., Saini, T. D., Starobinsky, A. A., \& Alam, U. 2003, JETP Lett., 77, 201

Sandvik, H., Tegmark, M., Zaldarriaga, M., \& Waga, I. 2002 [astro-ph/0212114]

Sarbu, N., Rusin, D., \& Ma, C.-P. 2001, ApJ, 561, L147

Schechter, P. L. 1976, ApJ, 203, 297

Silva, P. T., \& Bertolami, O. 2003 [astro-ph/0303353]

Spergel, D. N., et al. 2003 [astro-ph/0302209]

Turner, E. L., Ostriker, J. P., \& Gott, J. R. 1984, ApJ, 284, 1

Turner, E. L. 1990, ApJ, 365, L43

Watterich, C. 2002, Phys. Rev. D, 65, 123512

Zhu, Z.-H. 1998, A\&A, 338, 777

Zhu, Z.-H. 2000a, Mod. Phys. Lett. A, 15, 1023

Zhu, Z.-H. 2000b, Int. J. Mod. Phys. D, 9, 591 